\title{
Normative references of heart rate variability and salivary alpha-amylase in a healthy young male population
}

\author{
Hiromitsu Kobayashi ${ }^{1 *}$, Bum-Jin Park² and Yoshifumi Miyazaki ${ }^{3}$
}

\begin{abstract}
Background: This study aimed to present normative reference values of heart rate variability and salivary alphaamylase in a healthy young male population with a particular focus on their distribution and reproducibility.

Methods: The short-term heart rate variability of 417 young healthy Japanese men was studied. Furthermore, salivary alpha-amylase was measured in 430 men. The average age of the subjects were 21.9 years with standard deviation of 1.6 years. Interindividual variations in heart rate variability indices and salivary alpha-amylase levels were plotted as histograms. Data are presented as the mean, median, standard deviation, coefficient of variation, skewness, kurtosis, and fifth and 95th percentiles of each physiological index.

Results: Mean recorded values were heart period 945.85 ms, log-transformed high frequency component $9.84 \mathrm{ln}$ $\mathrm{ms}^{2}$, log-transformed low frequency component $10.42 \mathrm{ln}$ - $\mathrm{ms}^{2}$, log-transformed low frequency to high frequency ratio 0.58 In-ratio, standard deviation of beat-to-beat interval $27.17 \mathrm{~ms}$ and root mean square of successive difference $37.49 \mathrm{~ms}$. The mean value of raw salivary alpha-amylase was $17.48 \mathrm{U} / \mathrm{mL}$, square root salivary alphaamylase $3.96 \mathrm{sqrt}[\mathrm{U} / \mathrm{mL}]$ and log-transformed salivary alpha-amylase $2.65 \mathrm{In}[\mathrm{U} / \mathrm{mL}]$. Log-transformed heart rate variability indices exhibited almost symmetrical distributions; however, time-domain indices of heart rate variability (standard deviation of beat-to-beat interval and root mean square of successive difference) exhibited right-skewed (positive skewness) distributions. A considerable right-skewed distribution was observed for raw salivary alphaamylase. Logarithmic transformation improved the distribution of salivary alpha-amylase, although square root transformation was insufficient. The day-to-day reproducibility of these indices was assessed using intraclass correlation coefficients. Intraclass correlation coefficients of most heart rate variability and salivary indices were approximately 0.5 to 0.6 . Intraclass correlation coefficients of raw salivary markers were approximately 0.6 , which was similar to those of heart rate variability; however, log transformation of the salivary markers did not considerably improve their reproducibility. Correlations between sympathetic indicators of heart rate variability and salivary alpha-amylase were not observed.
\end{abstract}

Conclusion: Because the sample population examined in this study involved limited age and gender variations, the present results were independent of these factors and were indicative of pure interindividual variation.

Keywords: heart rate variability, kurtosis, reproducibility, salivary alpha-amylase, skewness

\footnotetext{
* Correspondence: kobayasi@ishikawa-nu.ac.jp

'Department of Nursing, Ishikawa Prefectural Nursing University, Ishikawa,

Japan

Full list of author information is available at the end of the article
} 


\section{Background}

Heart rate variability (HRV) has been extensively studied as an index of human autonomic nervous function. Low frequency (LF) and high frequency (HF) components of HRV are considered markers of sympathetic and parasympathetic nervous activities, respectively [1]. A relationship between HRV and autonomic functions has been established by several studies [2-4].

HRV measurements can be divided into two methods: long-term and short-term measurements. In long-term HRV measurements, heart rate is typically continuously recorded for 12 to 24 hours. Because it is a nonrestrained recording, subjects can continue their routine life during the measurement. Therefore, larger populations are relatively easy to evaluate in long-term HRV measurements, and some studies performed measurements in sample sizes of 1,000 individuals [5-7]. In contrast, 2- to 5-minute recordings are commonly used to measure short-term HRV [1]. In ambulatory measurements, a participant's body movements, utterances or other behaviors affect HRV. In particular, changes in posture and breathing can distinctly influence HRV $[8,9]$, decreasing the reliability of long-term HRV measurements [10]. In short-term measurements, these artifacts are controllable. However, the sample size tends to be smaller in short-term HRV measurements because these measurements are typically performed in laboratory settings.

In recent years, salivary alpha-amylase (sAA) has attracted attention as a biomarker of sympathetic nervous activity [11]. Amylase is an enzyme related to carbohydrate digestion, and its secretion is controlled by the sympathetic-adrenal medullary system. Similar to HRV measurements, sAA can be measured non-invasively. Recent developments in the devices for monitoring sAA have extended the application of this measurement into various fields of human science.

The results of HRV and SAA measurements indicate significant individual variations. Because of these variations, these physiological measurements often produce unclear results. Therefore, understanding individual variations in these physiological indices is necessary for appropriate interpretation of human autonomic nervous function. This study aimed to elucidate normative reference values of HRV and sAA in a healthy young male population with a special focus on their distribution and day-to-day reproducibility.

\section{Methods}

\section{Participants}

The study consisted of 456 Japanese male students aged 20 to 29 years old (mean, $21.9 \pm 1.6$ years). Height was in the range of 158 to $182 \mathrm{~cm}$ (mean $172.1 \pm 5.6 \mathrm{~cm}$ ) and weight was between 53 and $82 \mathrm{~kg}$ (mean $64.5 \pm 9.3$ $\mathrm{kg}$ ). All participants were non-smokers, and alcohol intake on the day before the measurement was forbidden.

The study was conducted under the regulations of the Institutional Ethical Committee of the Forestry and Forest Products Research Institute and Center for Environment, Health and Field Sciences, Chiba University, Japan. The aim and procedure of the experiment were explained to the participants, and written informed consent was obtained.

Measurements were performed in the morning before breakfast (6:30 to 7:30 a.m.). Each participant rested for 1 minute in a sitting position, and beat-to-beat intervals during spontaneous breathing were recorded for the next 2 minutes. The measurement was repeated using the same procedure one day after the first measurement to confirm intra-individual reproducibility. We measured HRV and sAA in 456 individuals; however, because of failed measurements, only 417 and 430 subjects were included in HRV and sAA analyses, respectively.

\section{Heart rate variability measurements}

Beat-to-beat heart rate was recorded with 1-ms resolution using portable heart rate monitors (AC301; GMS, Japan). Beat detection errors or any ectopic beats were corrected or excluded from the analysis. The heart period (HP), standard deviation of normal-to-normal intervals (SDNNs) and root mean square of successive differences (rMSSDs) were calculated from the 2-minute recordings.

Spectral analysis was performed by the maximum entropy method (MEM), and autoregressive coefficients were estimated using the Burg algorithm. A fixed autoregressive model order (12th) was used for the spectral analyses according to previous studies $[12,13]$. The power spectrum was calculated from 0.01 to $0.40 \mathrm{~Hz}$ with $0.01-\mathrm{Hz}$ frequency resolution. HF and LF components were calculated through integration of the power spectra of 0.15 to $0.40 \mathrm{~Hz}$ and 0.04 to $0.15 \mathrm{~Hz}$, respectively. HF, LF and the LF to HF ratio were then converted into natural logarithms (lnHF, $\operatorname{lnLF}$, and $\ln [\mathrm{LF} /$ $\mathrm{HF}])$.

\section{Salivary alpha-amylase measurement}

sAA was measured using a hand-held amylase monitor ( $\alpha$-AMY; YAMAHA, Japan). This device quantifies sAA activity based on a dry chemical system. The effects of environmental temperature and $\mathrm{pH}$ on individual samples were automatically adjusted to reflect the effects of $37^{\circ} \mathrm{C}$ and $\mathrm{pH} 6.5$. The accuracy of the amylase monitor has been validated previously [14]. Saliva samples from 
each individual were collected using a test strap. The time required for saliva collection using the strap was 2 minutes. The collected samples were immediately analyzed, and analysis was completed within a minute for each sample.

\section{Statistical analysis}

The average of the results from the two experimental days was used for analysis of distribution characteristics. The mean, median, SD, coefficient of variation (CV; SD/ mean), skewness (a measure of symmetry), kurtosis (a measure of peakedness) and fifth and 95th percentiles of the physiological indices were calculated.

Intraclass correlation coefficients (ICCs) were calculated from the results of two repeated measurements as an index of intra-individual reproducibility. Strictly speaking, ICC used in this study was ICC $(1,1)$ according to the classification by Shrout and Fleiss [15].

\section{Results}

The histograms of interindividual variations in HP and log-transformed HRV indices are shown in Figure 1. HP and log-transformed HRV indices exhibited symmetrical distributions; however, positive kurtosis (a slightly peaked curve) was observed for the distribution of $\ln$ (LF/HF) compared with the normal distribution. Regarding time-domain indices (SDNN and rMSSD), their skewness and kurtoses were high, and their distributions were considerably different from the normal distribution.

The mean, median, $\mathrm{SD}, \mathrm{CV}$, skewness, kurtosis and 5th and 95th percentiles of HRV indices are presented in Table 1. Interindividual variations (CVs) in the logtransformed HRV indices (lnHF and lnLF) were 7\% to 9\%; however, the time-domain indices (SDNN and rMSSD) exhibited high variabilities of $43 \%$ to $44 \%$.

Histograms of interindividual variations in raw and numerically transformed sAA values are shown in Figure 2. Extremely skewed distributions were observed for raw sAA values; square root and logarithmic transformations improved the skewness. The distribution characteristics of sAA are summarized in Table 2. Raw sAA values exhibited a large interindividual variation of approximately $67 \%$, and square root and natural logarithmic transformations diminished this variation to approximately $20 \%$ to $30 \%$. Numerical transformations

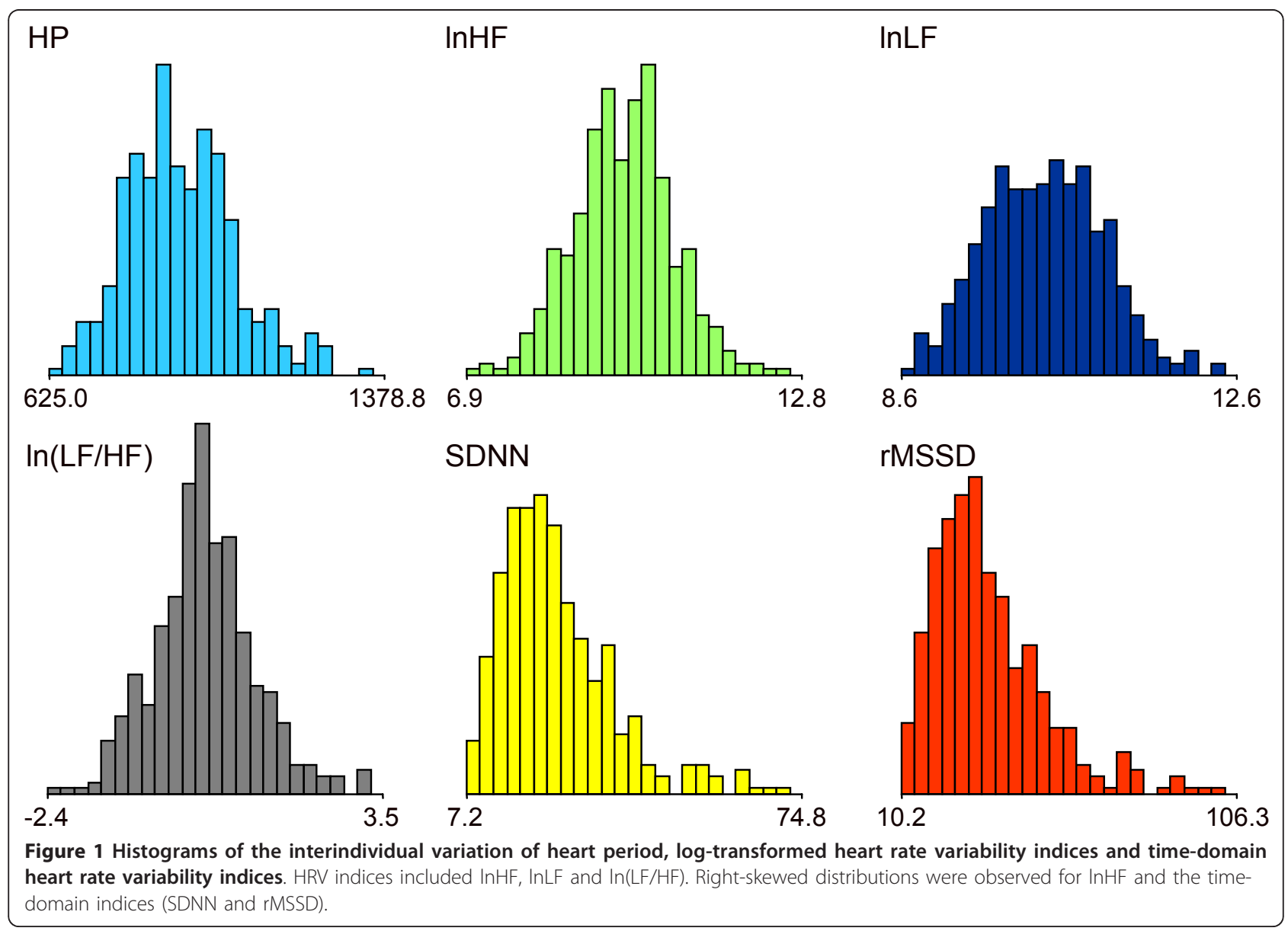


Table 1 Distribution characteristics of heart rate variability indices

\begin{tabular}{|c|c|c|c|c|c|c|}
\hline & $\begin{array}{l}\text { Heart period } \\
\text { (ms) }\end{array}$ & $\begin{array}{l}\text { InHF } \\
\left(\ln -\mathrm{ms}^{2}\right)\end{array}$ & $\begin{array}{l}\text { InLF } \\
\left({ }^{\prime n}-\mathrm{ms}^{2}\right)\end{array}$ & $\begin{array}{l}\text { In(LF/HF) } \\
\text { (In-ratio) }\end{array}$ & $\begin{array}{l}\text { SDNN } \\
\text { (ms) }\end{array}$ & $\begin{array}{l}\text { rMSSD } \\
\text { (ms) }\end{array}$ \\
\hline n & 417 & 417 & 417 & 417 & 417 & 417 \\
\hline mean & 945.85 & 9.84 & 10.42 & 0.58 & 27.17 & 37.49 \\
\hline median & 938.86 & 9.89 & 10.42 & 0.56 & 25.09 & 34.76 \\
\hline Standard deviation $^{\mathrm{a}}$ & 126.98 & 0.92 & 0.73 & 0.92 & 11.78 & 16.54 \\
\hline Coefficient of variation (\%) & 13.42 & 9.35 & 7.01 & $-\mathbf{b}$ & 43.36 & 44.12 \\
\hline Skewness & 0.38 & 0.01 & 0.11 & 0.19 & 1.22 & 1.24 \\
\hline Kurtosis & 0.26 & 0.32 & -0.21 & 0.71 & 1.91 & 2.04 \\
\hline Fifth percentile & 748.53 & 8.39 & 9.24 & -0.90 & 12.4 & 16.98 \\
\hline $95^{\text {th }}$ percentile & 1171.68 & 11.37 & 11.58 & 2.12 & 50.99 & 69.95 \\
\hline
\end{tabular}

HF: high frequency component; LF: low frequency component; rMSSD: root mean square of successive differences; SDNN: standard deviation of normal-to-normal intervals. Skewness is a measure of symmetry of distribution. Negative or positive skewness is indicated when the left or right tail of the histogram is longer, respectively. The skewness of a normally distributed data set is zero. Kurtosis is a measure of whether the distribution curve is peaked (positive) or flat (negative) relative to the normal distribution. The kurtosis of a normally distributed data set is zero. ${ }^{\mathrm{a}}$ Standard deviation of interindividual variation; ${ }^{\mathrm{b}}$ the coefficient of variation of $\ln (\mathrm{LF} / \mathrm{HF})$ was not calculated because it was occasionally negative.

also improved skewness and kurtosis; however, logarithmic transformation produced a better result (approximately equivalent to normal distribution), and square root transformation appeared insufficient.

The day-to-day reproducibility (ICCs) of HRV and salivary biomarker measurements are shown in Table 3 . The ICC of HP was 0.67, whereas that of most HRV indices was approximately 0.5 to 0.6 . Similar to the results for HRV, ICCs of raw and transformed sAA values were approximately 0.6 . In contrast with skewness and kurtosis, the reproducibility of sAA measurements was not considerably improved by numerical transformations.

Correlations between sympathetic indicators of HRV and log-transformed sAA measurements are presented in Figure 3. We analyzed 405 participants for whom HRV and sAA were successfully measured. Neither $\operatorname{lnLF}$ nor $\ln (\mathrm{LF} / \mathrm{HF})$ correlated with log-transformed sAA values.

\section{Discussion}

\section{Distribution characteristics of heart rate variability}

Some measurements of short-term HRV in large populations have been reported; however, only a few studies focused on the interindividual distribution of these measurements. The study by Kuo et al. [7] is one of the few studies that reported the distribution characteristics of HRV through histograms. In addition, they demonstrated that time-domain indices exhibited similar skewed distributions; our results were consistent with these findings. In our results, positive skewness was observed in the distributions of all indices. In contrast, Kuo et al. [7] reported slight negative skewness in lnHF and $\operatorname{lnLF}$ of Taiwanese men and women aged 40 to 79 years old. The difference in this distribution might be attributed to the differences in the age and sex of the sample populations.

Many studies reported that HRV is influenced by age and sex. Both HF and LF components decrease with

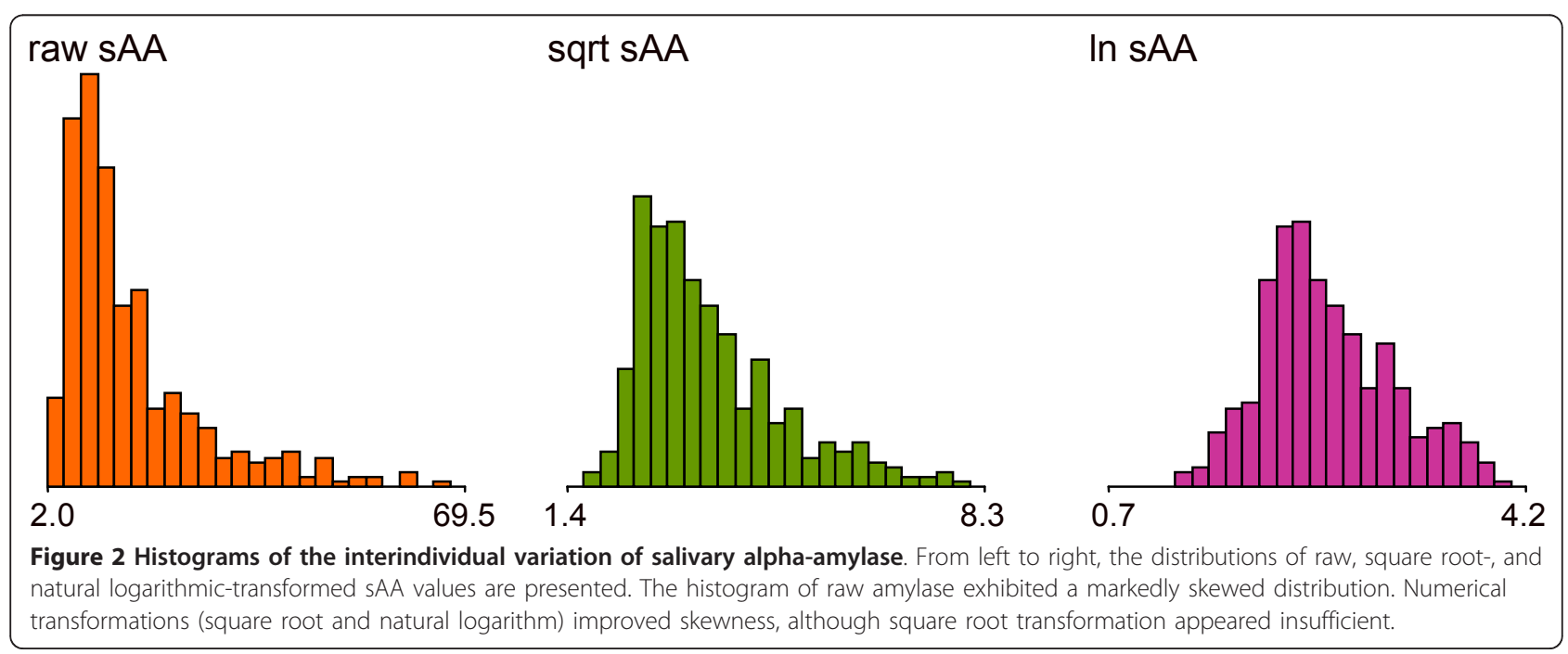


Table 2 Distribution characteristics of raw and numerically transformed salivary alpha-amylase values

\begin{tabular}{llll}
\hline & Raw sAA & \multicolumn{2}{c}{ Transformed sAA } \\
\cline { 3 - 4 } & $(\mathbf{U} / \mathbf{m L})$ & $\begin{array}{l}\text { Square root } \\
\text { (sqrt [U/mL]) }\end{array}$ & $\begin{array}{l}\text { Natural log } \\
(\mathbf{I n}[\mathbf{U} / \mathbf{m L}])\end{array}$ \\
\hline $\mathrm{n}$ & 430 & 430 & 430 \\
Mean & 17.48 & 3.96 & 2.65 \\
Median & 13.00 & 3.60 & 2.55 \\
Standard deviation & 11.70 & 1.21 & 0.57 \\
Coefficient of variation (\%) & 66.94 & 30.56 & 21.51 \\
Skewness & 1.79 & 1.11 & 0.31 \\
Kurtosis & 3.27 & 1.01 & 0.16 \\
Fifth percentile & 6.50 & 2.48 & 1.79 \\
95 & 43.75 & 6.06 & 3.71 \\
\hline
\end{tabular}

age, and men have relatively higher sympathetic predominance than women $[7,16]$. The effect of age and sex cannot be examined in our results because this study only examined healthy young men aged 20 to 29 years old.

The distributions of time-domain indices (SDNN and rMSSD) were far from normal. Compared with those in log-transformed HRV, interindividual variations in these indices were also extremely high. Therefore, when analyzing these indices, employing non-parametric tests or performing logarithmic transformation of the spectral components of HRV appears to be necessary.

\section{Distribution characteristics of salivary alpha-amylase}

Salivary biomarkers such as amylase or cortisol can exhibit skewness in their distributions. Therefore, these values are often analyzed after logarithmic transformation. Gordis et al. [17] reported that the skewness for cortisol was greater than that for sAA. In according with their result, some researchers performed logarithmic transformation for cortisol and square root transformation for amylase [18,19]. In our results, the distribution curve of amylase exhibited considerable skewness. Both square root and logarithmic transformations improved the skewness of sAA; however, better results were obtained with logarithmic transformation. Square root transformation appeared insufficient.

The study by Gordis et al. [17] was conducted on children, whereas the present study was conducted on young men. The difference in age of the two sample populations might explain the different distributions of sAA. Further investigations are expected on the distribution of the salivary biomarker.

\section{Limitations for the reference values of heart rate variability and salivary alpha-amylase}

The present results on HRV were calculated by MEM and thus the results are not comparable with other results calculated by fast Fourier transformation. However, the dimensionless indices such as $\mathrm{CV}$, skewness and kurtosis of the distribution might be comparable with other results even if they were calculated by fast Fourier transformation.

HRV was measured during spontaneous breathing, and paced breathing was not applied in this study. Previous studies [12,20-22] reported that the LF component is decreased by paced breathing. Thus, lower LF values will be expected in the results measured during paced breathing compared with the results of this study. Paced breathing may also affect the interindividual distribution of HRV. However, a previous study reported that the effect of paced breathing on interindividual variations in the spectral components of HRV was negligible [22].

The presented values of sAA were small compared with the values reported by other studies. sAA represents apparent diurnal changes because its levels are lower in the early morning $[23,24]$. Raw (non-numeric transformed) sAA levels in the afternoon have been reported to be two- to three-fold higher than those in the early morning. sAA measurements of this study were performed in the early morning before breakfast. Thus, the difference is partly attributed to the time of sAA measurement. It is critical to note the time of day that sAA was measured when quantitatively examining the sAA values of different studies.

Table 3 Day-to-day reproducibility of heart rate variability and salivary alpha-amylase

\begin{tabular}{llc}
\hline & $\mathbf{n}$ & Intraclass correlation coefficients \\
\hline Heart period & 417 & 0.67 \\
InHF & 417 & 0.59 \\
InLF & 417 & 0.50 \\
In(LF/HF) & 417 & 0.54 \\
SD of normal-to-normal intervals & 417 & 0.56 \\
Root mean square of successive differences & 417 & 0.58 \\
Raw sAA & 430 & 0.56 \\
Square root transformed sAA & 430 & 0.61 \\
Natural log-transformed sAA & 430 & 0.60 \\
\hline
\end{tabular}

HF: high frequency component; LF: low frequency component; sAA: salivary alpha-amylase. 




In this study, sAA was measured using a hand-held amylase monitor, and the saliva samples were analyzed immediately after saliva collection. Conversely, in most previous studies on sAA [25-28], collected saliva was frozen and stored before biochemical analysis. This difference in the measurement procedure might affect the results of sAA measurement, although it has been reported that freezing and thawing does not significantly affect sAA [18]. Further study on the effect of the device and procedure of sAA measurement is needed.

\section{Reproducibility of heart rate variability and salivary alpha-amylase measurements}

One of the features of this study was the use of repeated measurements on different days. This permitted the analysis of the reproducibility of each HRV and salivary index. The present study appears to be the largest data set used to examine the reproducibility of short-term HRV measurements. The reproducibility (ICCs) of HRV measurements obtained in this study was slightly lower than that obtained in previous studies $[12,20,21]$. This may have resulted from the small age variation among the participants of this study. As mentioned previously, HRV is affected by age and thus interindividual variation in HRV decreases when age variation is narrow. A study of HRV in Japanese men aged 20 to 61 years old reported $14 \%$ to $16 \%$ interindividual variations in $\operatorname{lnHF}$ and $\operatorname{lnLF}$ [29]; conversely, the variations were $7 \%$ to $9 \%$ in this study. Roughly interpreted, ICC is the ratio of interindividual variation to total variation; therefore,
ICC tends to be low when the interindividual variation is low [30].

Compared with that of HRV, the day-to-day reproducibility of sAA is not well known. Very high reproducibility (ICC $=0.92$ ) of sAA measured using a hand-held monitor was recently reported by Aoyagi et al. [31]. However, sAA measurements were consecutively repeated in that study and thus did not reflect day-today reproducibility. Wolf et al. [27] reported that ICC of sAA measurements was 0.5 to 0.7 when the measurements were repeated within a span of several hours. Their result is similar to ours, although the details of the measurements were different.

\section{Correlation between sympathetic indicators of heart rate variability and salivary alpha-amylase}

The previous studies that demonstrated a relationship between sAA and sympathetic activity (plasma norepinephrine level) examined responses to physical or mental stress [23,32], whereas the present study measured sympathetic indicators of a homogeneous population under resting condition.

If sAA is an indicator of sympathetic nervous activity, some correlation with HRV should be expected regardless of whether under resting conditions or responses to stress. In the present study, however, no correlations were observed between sAA and HRV. The interindividual variations of sAA and HRV under resting condition might not imply the variation in sympathetic tone but some other aspect of human physiology. 
In addition to our results, other previous studies have also demonstrated small or insignificant correlations between sAA and sympathetic markers of HRV, although they examined the variations including responses to the various kinds of stress $[11,25,27,33]$. We must note that there is room for discussion on the relationship between sAA and other sympathetic indicators.

\section{Conclusions}

Because the sample population examined in this study involved limited age and gender variations, the present results were independent of these factors and were indicative of pure interindividual variation. In other words, the interindividual variations presented in this study are the lower limit, and the variation in actual population will be larger than the values reported in this study.

\section{Acknowledgements}

This study was partially supported by Grants-in-Aid for Scientific Research (S; 16107007) from the Ministry of Education, Culture, Sports, Science and Technology, Japan.

\section{Author details}

${ }^{1}$ Department of Nursing, Ishikawa Prefectural Nursing University, Ishikawa, Japan. ${ }^{2}$ Department of Environment \& Forest Resources, Chungnam National University, Deajeon, Korea. ${ }^{3}$ Center for Environment, Health and Field Sciences, Chiba University, Chiba, Japan.

\section{Authors' contributions}

HK carried out the statistical analysis and interpretation of the results. BP carried out data collection and analysis. YM had overall responsibility for the study design. All authors read and approved the final manuscript.

\section{Competing interests}

The authors declare that they have no competing interests.

Received: 10 May 2011 Accepted: 20 April 2012 Published: 20 April 2012

\section{References}

1. Task Force of the European Society of Cardiology and the North American Society of Pacing and Electrophysiology: Heart rate variability - standards of measurement, physiological interpretation, and clinical use. Circulation 1996, 93:1043-65.

2. Akselrod S, Gordon D, Ubel FA, Shannon DC, Barger AC, Cohen RJ: Power spectrum analysis of heart rate fluctuation: a quantitative probe of beat to beat cardiovascular control. Science 1981, 213:220-222.

3. Malliani A, Pagani M, Lombardi F, Cerutti S: Cardiovascular neural regulation explored in the frequency domain. Circulation 1991, 84:1482-92.

4. Montano N, Ruscone TG, Porta A, Lombardi F, Pagani M, Malliani A: Power spectrum analysis of heart rate variability to assess the changes in sympathovagal balance during graded orthostatic tilt. Circulation 1994, 90:1826-31.

5. Liao D, Barnes RW, Chambless LE, Simpson RJ Jr, Sorlie P, Heiss G: Age, race, and sex differences in autonomic cardiac function measured by spectral analysis of heart rate variability - the ARIC study. Atherosclerosis Risk in Communities. Am J Cardiol 1995, 76:906-912

6. Tsuji H, Venditti FJ Jr, Manders ES, Evans JC, Larson MG, Feldman CL, Levy D: Determinants of heart rate variability. J Am Coll Cardiol 1996, 28(6):1539-1546.

7. Kuo TB, Lin T, Yang CC, Li CL, Chen CF, Chou P: Effect of aging on gender differences in neural control of heart rate. Am J Physiol 1999, 277: $\mathrm{H} 2233-\mathrm{H} 2239$
8. Kobayashi $\mathrm{H}$ : Postural effect on respiratory sinus arrhythmia with various frequencies. Appl Hum Sci 1996, 15:87-91.

9. Kobayashi H: Normalization of respiratory sinus arrhythmia by factoring in tidal volume. Appl Hum Sci 1998, 17:207-213.

10. Hartikainen JEK, Tahvanainen KUO, Kuusela TA: Short-term measurement of heart rate variability. In Clinical Guide to Cardiac Autonomic Tests. Edited by: Malik M. Dordrecht (Netherlands): Kluwer; 1998:149-176.

11. Nater UM, Rohleder N: Salivary alpha-amylase as a noninvasive biomarker for the sympathetic nervous system: current state of research. Psychoneuroendocrinology 2009, 34:486-496.

12. Pitzalis MV, Mastropasqua F, Massari F, Forleo C, Di Maggio M, Passantino A, Colombo R, Di Biase M, Rizzon P: Short- and long-term reproducibility of time and frequency domain heart rate variability measurements in normal subjects. Cardiovasc Res 1996, 32:226-233.

13. Fagard RH, Pardaens K, Staessen JA: Influence of demographic, anthropometric and lifestyle characteristics on heart rate and its variability in the population. J Hypertens 1999, 17:1589-1599.

14. Yamaguchi M, Kanemori T, Kanemaru M, Takai N, Mizuno Y, Yoshida H: Performance evaluation of salivary amylase activity monitor. Biosens Bioelectron 2004, 20:491-497.

15. Shrout PE, Fleiss JL: Intraclass correlations: uses in assessing rater reliability. Psychol Bull 1998, 7:301-317.

16. Agelink MW, Malessa T, Baumann B, Majewski T, Akila F, Zeit T, Ziegler D: Standardized tests of heart rate variability: normal ranges obtained from 309 healthy humans, and effects of age, gender and heart rate. Clinic Auton Res 2011, 11:99-108.

17. Gordis EB, Granger DA, Susman EJ, Trickett PK: Asymmetry between salivary cortisol and alpha-amylase reactivity to stress: relation to aggressive behavior in adolescents. Psychoneuroendocrinology 2006, 31:976-987.

18. Granger DA, Kivlighan KT, el-Sheikh M, Gordis EB, Stroud LR: Salivary alphaamylase in biobehavioral research: recent developments and applications. Ann N Y Acad Sci 2007, 1098:122-144

19. Fortunato CK, Dribin AE, Granger DA, Buss KA: Salivary alpha-amylase and cortisol in toddlers: differential relations to affective behavior. Dev Psychobiol 2008, 50:807-818.

20. Carrasco S, González R, Gaitán MJ, Yáñez O: Reproducibility of heart rate variability from short-term recordings during five maneuvers in normal subjects. J Med Eng Technol 2003, 27:241-248.

21. Reland S, Ville NS, Wong S, Carrault G, Carré F: Reliability of heart rate variability in healthy older women at rest and during orthostatic testing. Aging Clin Exp Res 2005, 17:316-321.

22. Kobayashi $\mathrm{H}$ : Does paced breathing improve the reproducibility of heart rate variability measurements? J Physiol Anthropol 2009, 28:225-230.

23. Rohleder N, Nater UM, Wolf JM, Ehlert U, Kirschbaum C: Psychosocial stress-induced activation of salivary alpha-amylase: an indicator of sympathetic activity? Ann N Y Acad Sci 2004, 1032:258-263.

24. Nater UM, Rohleder N, Schlotz W, Ehlert U, Kirschbaum C: Determinants of the diurnal course of salivary alpha-amylase. Psychoneuroendocrinology 2007, 32:392-401.

25. Granger DA, Blair C, Willoughby M, Kivlighan KT, Hibel LC, Fortunato CK, Wiegand LE: Individual differences in salivary cortisol and alpha-amylase in mothers and their infants: relation to tobacco smoke exposure. Dev Psychobiol 2007, 49:692-701.

26. Nater UM, La Marca R, Florin L, Moses A, Langhans W, Koller MM, Ehlert U: Stress-induced changes in human salivary alpha-amylase activity associations with adrenergic activity. Psychoneuroendocrinology 2006 31:49-58.

27. Wolf JM, Nicholls E, Cheen E: Chronic stress, salivary cortisol, and alphaamylase in children with asthma and healthy children. Biol Psychol 2008, 78:20-28.

28. Filaire $E$, Portier $H$, Massart A, Ramet $L$, Teixeira A: Effect of lecturing to 200 students on heart rate variability and alpha-amylase activity. Eur J Appl Physiol 2010, 108:1035-1043.

29. Kobayashi $\mathrm{H}$ : Inter- and intra-individual variations of heart rate variability in Japanese males. J Physiol Anthropol 2007, 26:173-177.

30. Weir JP: Quantifying test-retest reliability using the intraclass correlation coefficient and the SEM. J Strength Cond Res 2006, 19:231-240.

31. Aoyagi K, Karibe H, Kawakami T, Warita S, Shimazu K, Ogata K: Inter- and intraobserver reliability of the hand-held monitor for measuring salivary alpha-amylase level. Clin Lab 2011, 57:253-257. 
32. Chatterton RT, Vogelsong KM, Lu YC, Ellman AB, Hudgens GA: Salivary alpha-amylase as a measure of endogenous adrenergic activity. Clin Physiol 1996, 16(4):433-448.

33. Rohleder N, Wolf JM, Maldonado EF, Kirschbaum C: The psychosocial stress-induced increase in salivary alpha-amylase is independent of saliva flow rate. Psychophysiology 2006, 43:645-652.

doi:10.1186/1880-6805-31-9

Cite this article as: Kobayashi et al: Normative references of heart rate variability and salivary alpha-amylase in a healthy young male population. Journal of Physiological Anthropology 2012 31:9.

Submit your next manuscript to BioMed Central and take full advantage of:

- Convenient online submission

- Thorough peer review

- No space constraints or color figure charges

- Immediate publication on acceptance

- Inclusion in PubMed, CAS, Scopus and Google Scholar

- Research which is freely available for redistribution

Submit your manuscript at www.biomedcentral.com/submit 